Original Paper http://ajol.info/index.php/ijbcs http://indexmedicus.afro.who.int

\title{
Conduite de l'élevage au sol des poules pondeuses : cas des fermes avicoles de Niamey et Tillabéri en République du Niger
}

\author{
Harouna ABDOU ${ }^{1 *}$, Abdoulkadri LAOUALI ${ }^{2}$ et Balkissa ROUGA ASSOUMANE ${ }^{1}$ \\ ${ }^{I}$ Université Boubacar BÂ de Tillabéri, Faculté des Sciences Agronomiques, Département des Productions \\ Animales, BP : 175 Tillabéri-Niger. \\ ${ }^{2}$ Université Boubacar BÂ de Tillabéri, Faculté des Sciences Agronomiques, Département Socio-économie, \\ BP : 175 Tillabéri-Niger. \\ *Auteur correspondant ; E-mail : bassarou74@ gmail.com; Tél : +22796038018/+22780806518
}

\section{RÉSUMÉ}

L'objectif général de ce travail est d'étudier les pratiques de l'élevage au sol des poules pondeuses. La zone d'étude s'étend entre les régions de Niamey et Tillabéri (Niger). Au total, dix (10) fermiers avicoles pratiquant l'élevage au sol des poules pondeuses dont neuf (9) à Niamey et un (1) à Tillabéri ont été choisis. La pratique de l'élevage au sol des poules pondeuses est retenue comme critère d'inclusion pour l'échantillonnage. La collecte des données a été possible par l'administration des questionnaires qui s'est effectuée à travers des entretiens individuels. L'étude révèle que la majorité des fermiers sont des adultes avec un âge moyen qui tourne autour de 50 (donné l'écart type) ans. Une majeure partie (80\%) des répondants était instruite. L'échantillon est composé de $70 \%$ de fonctionnaires contre $30 \%$ de commerçants. Il est observé au cours de cette étude, que $70 \%$ des fermes de l'échantillon ne renferment que 1 à 2 bâtiments et $30 \%$ qui disposent entre 3 à 5 bâtiments. L'analyse des données a permis de constater que $20 \%$ de ces fermes avicoles ont une superficie comprise entre 100 à $140 \mathrm{~m}^{2}$ tandis que $80 \%$ s'étendent sur une aire comprise entre 140 à $150000 \mathrm{~m}^{2}$. Par ailleurs, il ressort que $90 \%$ de l'échantillon de la présente étude achètent l'aliment complet préparé. Par contre, 10\% achètent les ingrédients et préparent l'aliment selon les normes à incorporer. Il est apparu au cours de cette enquête que $90 \%$ des enquêtés pratiquent périodiquement la désinfection des matériels. Ils appliquent la même méthode de désinfection et le même type de produit (crésyl et eau de javel). Au regard de ces résultats préliminaires, les conditions d'élevage au sol des poules pondeuses nécessitent une amélioration. Pour cela, la présente suggère d'autres investigations dans l'avenir.

(C) 2020 International Formulae Group. All rights reserved.

Mots clés : Etude, Pratiques, Elevage au sol, Poules pondeuses, Niger.

\section{Management of laying hens on the ground: case of the poultry farms of Niamey and Tillabéri in the Republic of Niger}

\begin{abstract}
He general objective of this work is to study the practices of floor rearing of laying hens. The study area extends between the regions of Niamey and Tillabéri (Niger). A total of ten (10) poultry farmers practicing ground rearing of laying hens were selected, including nine (9) in Niamey and one (1) in Tillabéri. Ground rearing
\end{abstract}


of laying hens was selected as a sampling criterion. Data collection was made possible by the administration of questionnaires through individual interviews. The study reveals that the majority of farmers are adults with an average age of around 50 (given the standard deviation) years. Major parts (80\%) of the respondents were educated. The sample is composed of $70 \%$ civil servants against $30 \%$ traders. It is observed during this study that $70 \%$ of the farms in the sample have only 1 to 2 buildings and $30 \%$ have 3 to 5 buildings. Analysis of the data showed that $20 \%$ of these poultry farms have an area of between 100 and $140 \mathrm{~m}^{2}$, while $80 \%$ have an area of between 140 and $150,000 \mathrm{~m}^{2}$. In addition, it was found that $90 \%$ of the sample in this study purchased prepared complete feed. On the other hand, $10 \%$ purchase the ingredients and prepare the food according to the standards to be incorporated. During this survey, it appeared that $90 \%$ of the respondents periodically practice disinfection of equipment. They apply the same method of disinfection and the same type of product (cresyl and bleach). In view of these preliminary results, the conditions in which laying hens are reared on the ground need to be improved. For this purpose, the present report suggests further investigations in the future.

(C) 2020 International Formulae Group. All rights reserved.

Keywords: Study, Practices, Ground rearing, Laying hens, Niger.

\section{INTRODUCTION}

L'élevage est d'une grande importance socio-économique car occupe $80 \%$ de la population et fait vivre de façon exclusive $20 \%$ de la population nigérienne (CILSS et al., 2006; FAOSTAT, 2012). En Afrique subsaharienne en général et au Niger en particulier où la problématique de la sécurité alimentaire se trouve au cœur de tout projet de développement durable, l'aviculture se distingue comme une filière de survivance et de rente dynamique qu'il faut encourager (Ouedraogo, 2003). Elle est accessible aux populations les plus pauvres, et notamment aux femmes dans le cadre domestique. Le Niger compte plus de 38 millions de volailles domestiques dont la quasi-totalité est sous gestion traditionnelle (FAOSTAT, 2012). L'aviculture moderne qui fait intervenir des souches de volaille et des techniques d'élevage différentes de celles de l'aviculture villageoise (Issa et al., 2010), représente moins de 10\% de la production nationale. Au Niger, Les principales contraintes au développement de cette aviculture moderne sont les charges d'exploitation dues essentiellement à l'alimentation. En effet, l'alimentation représente $60-80 \%$ du cout de production du fait qu'elle est principalement basée sur des matières premières importées (Issa et al., 2010), alors même qu'elle constitue l'un des principaux facteurs de réussite dans une entreprise avicole.

Le développement de l'aviculture nécessite une disponibilité soutenue des matières premières, en l'occurrence des bâtiments bien équipés, les poussins d'un jour, les ressources alimentaires en quantité et qualité (Nworgu et Fasoghon, 2007; Houndonougbo et al., 2012b) et les soins qui conditionnent le développement des performances de production. Malheureusement, les problèmes liés à l'alimentation (Issa et al., 2010), à la santé de la volaille (Maikano et Alassane, 2006), au coût élevé de la production, à l'hygiène de l'habitat, à la non maîtrise des normes techniques et de la gestion des affaires par les aviculteurs, demeurent encore comme contraintes majeures dont souffre le secteur avicole au Niger. Il est clairement indiqué dans la littérature qu'au nombre des obstacles majeurs qui handicapent l'essor de l'aviculture en Afrique, figure l'insuffisance en alimentation (Dahouda et al., 2009). L'aviculture moderne connait une croissance remarquable au cours de la dernière décennie dans notre pays. Néanmoins, l'impulsion de cette filière souffre de l'accompagnement des pouvoirs publics. La question prégnante est celle de l'alimentation. D'ailleurs, on a coutume de dire qu'en production animale, 
l'alimentation est le nerf de la guerre. Il est dit également qu'elle représente la part importante du coût de la production (Dronne, 2019). Parmi les contraintes de la pratique de l'élevage avicole au sol, il est cité également la santé et l'habitat.

Bien que la problématique demeure sur les pratiques d'élevage au sol, à notre connaissance, peu d'études se sont intéressées à la thématique. Â la lumière de ce qui précède, il serait intéressant d'agir pour remédier à cette problématique. Le présent travail s'inscrit dans cette logique. Il s'agit de mener une investigation sur les pratiques (la santé, l'hygiène, l'alimentation et les conditions de logement) d'élevage au sol et apporter des corrections afin que ces élevages puissent produire des œufs de qualité et en quantité pour satisfaire les consommateurs. L'objectif général de ce travail est d'étudier les pratiques de l'élevage au sol des poules pondeuses au sein des différentes fermes enquêtées.

\section{MATERIEL ET METHODES}

\section{Présentation de la Zone d'étude}

La zone d'étude s'étend entre les régions de Niamey et Tillabéri. Au niveau de la région de Tillabéri, nous avons la commune urbaine de Tillabéri (CUT) et pour celle de Niamey, nous avons retenu la communauté urbaine de Niamey (CUN) capitale politique du Niger (Figure 1) est situé entre $13^{\circ} 35$ de latitude Nord et $2^{\circ} 03$ et $2^{\circ} 10$ de longitude Est avec une superficie de $240 \mathrm{~m}^{2}$. Le climat est de type sahélien (semi-aride) avec une pluviométrie annuelle variant de 500 à 750 $\mathrm{mm}$. La CUT est située au sud-ouest de la République du Niger entre $14^{\circ} 12^{\prime} 37^{\prime \prime}$ Nord, et $1^{\circ} 27^{\prime} 10^{\prime \prime}$ Est (Figure 1). Le milieu physique de cette commune se caractérise par un relief constitué de plateaux et de collines. Le climat est de type sahélien où la température varie de 25,6 ${ }^{\circ} \mathrm{C}$ (minima) à $43{ }^{\circ} \mathrm{C}$ (maxima). Quant aux précipitions, la moyenne annuelle au cours de la dernière décennie est de $486 \mathrm{~mm}$ (INS, 2018). La CUT couvre une superficie de 97251 $\mathrm{km}^{2}$, soit 7,7\% du pays avec une population de 51439 habitants (MP-Niger, 2012) soit une densité de $28 \mathrm{hab}$. $/ \mathrm{km}$.

\section{Collecte des données}

Il s'agit d'un échantillon constitué de dix (10) fermiers avicoles qui pratiquent l'élevage au sol des poules pondeuses dont neuf (9) à Niamey et un (1) à Tillabéri. L'identification de ces neuf (9) fermes à Niamey a été possible grâce à l'appui des personnes ressources (professionnels du domaine). Le critère d'inclusion retenu pour le choix des fermes à enquêter a été la pratique de l'élevage au sol de poules pondeuses. Dans cette logique, des fiches ont été établis au préalable L'administration des questionnaires s'est effectuée à travers des entretiens individuels. Les grandes lignes du questionnaire sont: (1) les caractéristiques sociodémographiques des répondants; (2) les caractéristiques de l'habitat des poules pondeuses; (3) l'identification de type de ration formulée aux poules pondeuses; (4) évaluer la prophylaxie et les soins sanitaires mis au point.

\section{Traitement des données}

Après la collecte, les données ont été enregistrées dans le logiciel Excel avant d'être soumises à des analyses statistiques. Le logiciel SPSS a permis de déterminer pour chaque question, les proportions des répondants selon les réponses données. 


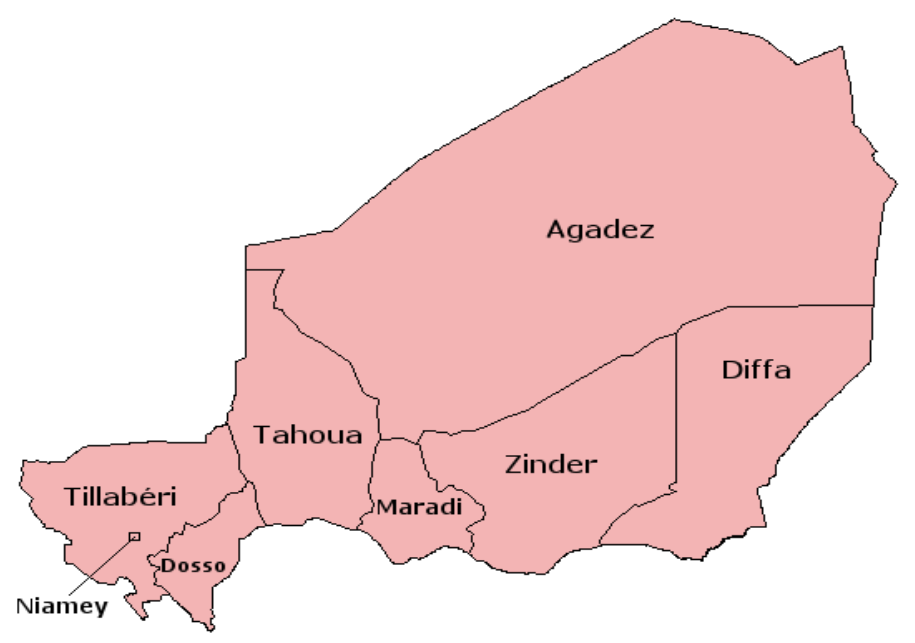

Figure 1: Localisation de la zone d'étude

Source :

https://www.google.com/search?sxsrf=ALeKk01hl1 VPFFveAG9XYAbhYxM_YzVZng:1587029497629\&q=Carte+administ rative+de+Niamey+et+Tillab\%C3\%A9ri\&tbm=isch\&source=univ\&client=firefoxd\&sa=X\&ved=2ahUKEwjF5NDL0ezoAh UxyIUKHTADBvcQsAR6BAgKEAE\&biw=1366\&bih=654\#imgrc=2TuVDISo1ylevM

\section{RESULTATS}

\section{Caractéristiques Sociodémographiques des aviculteurs}

Parmi les fermiers enquêtés $70 \%$ ont un âge compris entre 35 et 55 ans et $30 \%$ ont un âge qui compris entre 60 et 70 ans (Figure 2). Il ressort du présent travail que $80 \%$ des répondants ont fréquenté l'école par contre $20 \%$ de l'échantillon n'ont jamais été à l'école. L'enquête a permis de constater que $70 \%$ de l'échantillon sont des fonctionnaires $30 \%$ sont des commerçants. Par ailleurs, ils affirment que la production de leurs exploitations (œufs) est destinée à la vente, raison pour laquelle ils s'intéressent à l'aviculture. Pour le statut matrimonial, il ressort de cette étude que $100 \%$ des enquêtés sont des chefs de ménages (mariés).

Caractéristiques de l'habitat des poules pondeuses

Le nombre de bâtiment dans une ferme avicole est un indicateur clé pour apprécier la taille d'une entreprise agricole telle que la ferme avicole. L'enquête révèle que $70 \%$ des ne renferment que de 1 à 2 bâtiments. Seulement $30 \%$ de ces enquêtés disposaient de 3 à 5 bâtiments. Quant à la distance entre deux bâtiments, les fermiers (100\%) n'ont pas pris en compte cet aspect. Ils affirment n'avoir pas une connaissance sur les normes de superficies. L'analyse des données a permis de constater que $20 \%$ de ces fermes avicoles ont une superficie compris entre 100 et $140 \mathrm{~m}^{2}$ tandis que $80 \%$ s'étendent sur une superficie comprise entre 140 et $150000 \mathrm{~m}^{2}$ (Figure 3). Les bâtiments de tous les enquêtés (100\%) suivent la même direction Est-Ouest des vents dominants et ont la même nature de sol qui est en béton.-L'analyse des données révèle que $20 \%$ des répondants ont conçus leur bâtiment sans faitière et toit. À l'inverse, $80 \%$ des fermes ont été conçu avec toit et faitière. Les résultats montrent que $100 \%$ des répondants ont la ventilation naturelle ou statique, ils font le chauffage avec du charbon pour le démarrage des poussins, et l'éclairage se fait par la lumière naturelle et les lampes à panneaux solaire.

\section{Equipements d'élevages}

La Figure 4 présente les types d'abreuvoirs utilisés dans les fermes qui ont été suivi au cours du présent travail. Il ressort de cette étude que 50\% des répondants utilisent 
l'abreuvoir de type a (Figure 4a), 30\% font recours à l'abreuvoir de type b (Figure $4 \mathrm{~b}$ ) tandis que les abreuvoirs de type c (Figure 4c) et de type d (Figure 4d) sont utilisés dans les proportions équitables $(10 \%)$.

Les Figures $5 \mathrm{a}$ et $5 \mathrm{~b}$ présentent respectivement les mangeoires utilisées en phase démarrage-croissance et en phase de finition. Au terme de cette étude, il est constaté que toutes les fermes (100\%) utilisent les mêmes types de mangeoires et pondoir au cours des différentes étapes de la carrière reproductive de la poule pondeuse.

\section{Conduite d'alimentation}

Il ressort de cette étude que $90 \%$ de l'échantillon de la présente étude achètent l'aliment complet préparé. Par contre, $10 \%$ de cette population cible (échantillon) achètent les ingrédients et préparent l'aliment selon les normes à incorporer. Aucune de ces fermes n'a un atelier de transformation d'aliment. Ils distribuent tous l'aliment sous forme de miette. Le Tableau 1 présente la composition de l'aliment complet et celui préparé.

\section{Soins sanitaires et prophylactiques}

Il ressort de l'étude que $90 \%$ des enquêtés pratiquent périodiquement la désinfection des matériels. Ils appliquent la même méthode de désinfection et le même type de produit (crésyl, et eau de javel). Tous les fermiers $(100 \%)$ ne procèdent au contrôle visuel des animaux avant leurs installations qu'en cas de suspicion. Il est remarqué aussi que $40 \%$ des fermes ont des pédiluves à l'entrée du bâtiment et que $60 \%$ n'en disposent pas. Tous les répondants $(100 \%)$ affirment avoir un plan de prophylaxie médical, un référant sur le bien-être animal (technicien). Aucune exploitation (ferme) ne possède un espace dédié aux animaux malade (infirmerie).

Il ressort de cette étude que $100 \%$ des enquêtés ne font le contrôle de la litière qu'en cas de suspicion. Aucune de ces fermes ne dispose d'un espace réservé pour les cadavres des animaux. Il est observé que $20 \%$ font la différence entre les zones sale et propre

\section{Contraintes}

Tous les enquêtés (100\%) font face à de nombreuses difficultés dans la mesure où ils ne maitrisent pas le domaine à l'entame de cette activité d'aviculture. Quant aux techniciens (les vétérinaires), leurs décisions ne sont prises en compte que dans de rares cas.

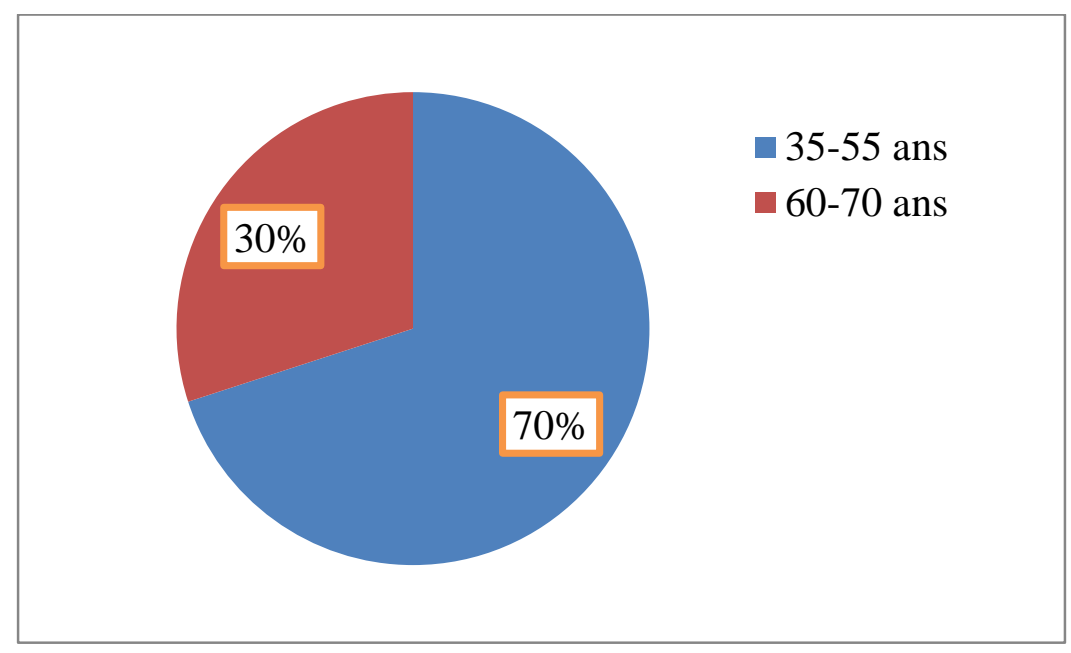

Figure 2: Répartition des répondants en fonction de l'âge. 
H. ABDOU et al. / Int. J. Biol. Chem. Sci. 14(3): 848-858, 2020

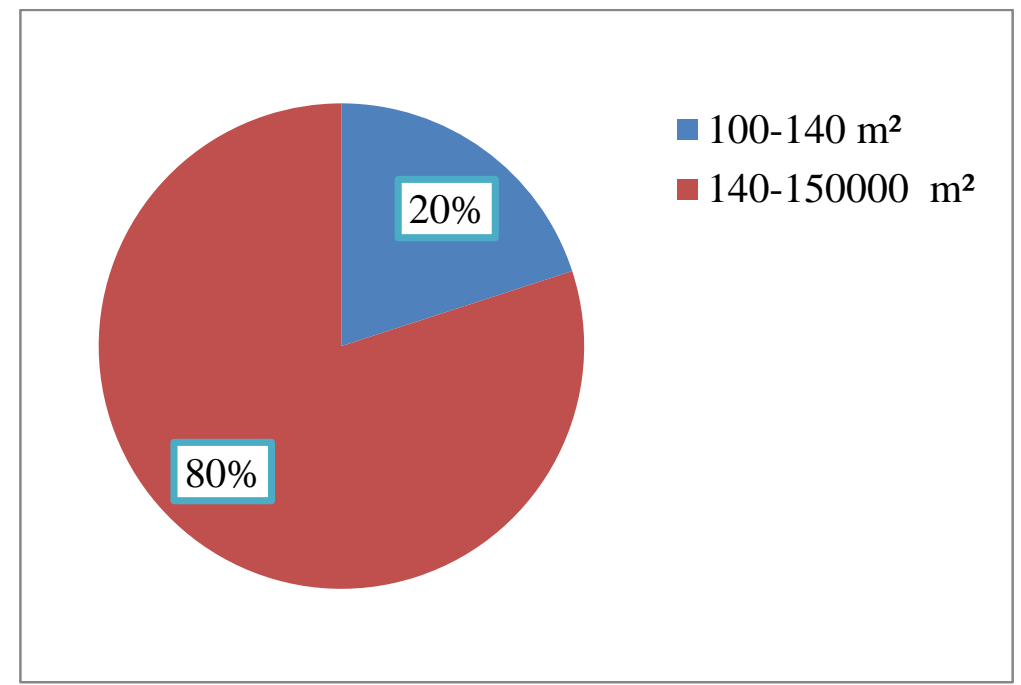

Figure 3: Dimensions des bâtiments.

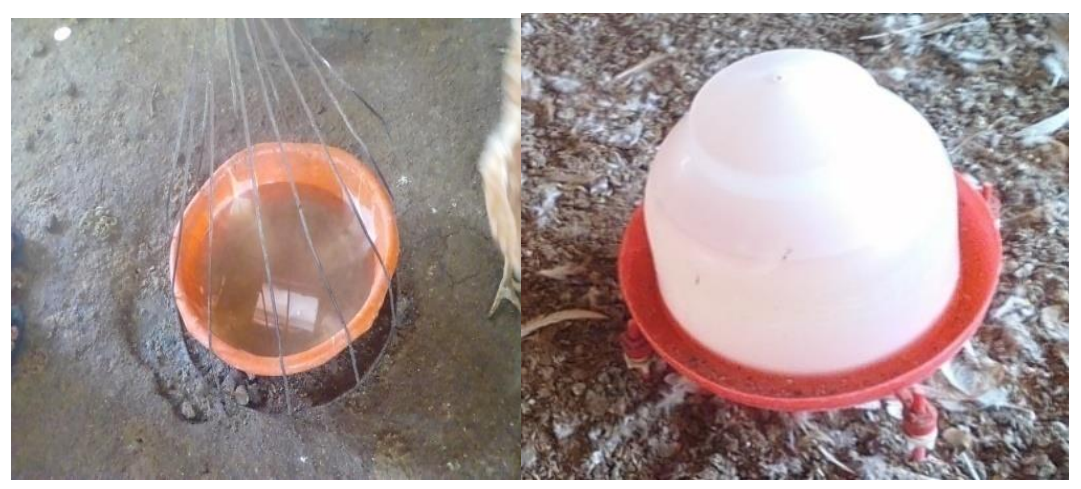

(a)

(b)

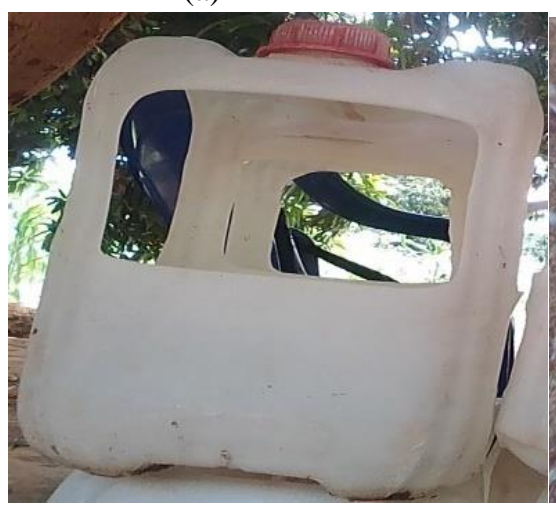

(c) (d)

Figure 4: Types d'abreuvoirs utilisés dans les fermes enquêtées. 


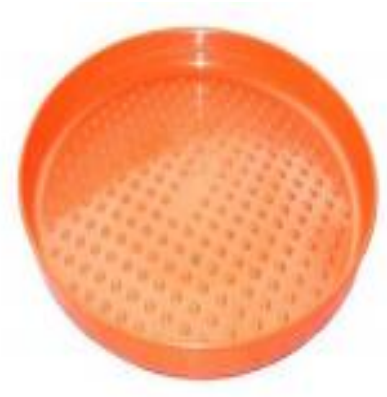

(a)

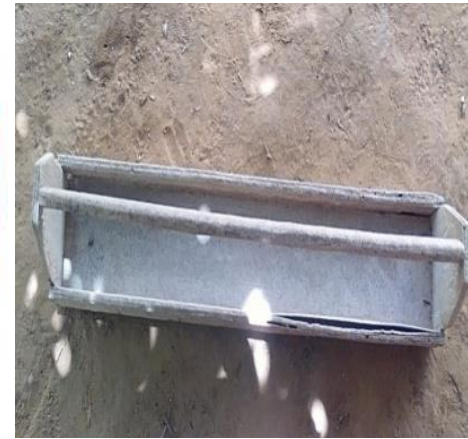

(b)

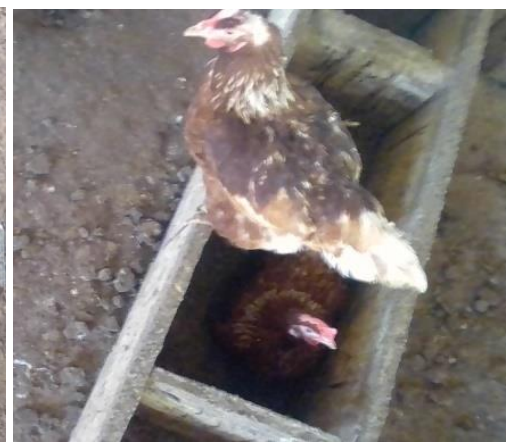

(c)

Figure 5: Types de mangeoires et pondoirs utilisés dans les fermes enquêtées.

Tableau 1: Composition de l'aliment complet et celui préparé utilisés dans les fermes enquêtés.

\begin{tabular}{ll}
\hline Aliment complet & Aliment préparé \\
\hline Composition & Composition \\
Céréales et issues & Son de blé \\
Sous-produits oléagineux & Son de maïs \\
19 Minéraux & Concentré \\
19 Acides aminés, sel & \\
Antioxydant & \\
Capteurs de mycotoxine & \\
Valeurs nutritionnelles & \\
Protéine & 15,8 \\
Lysine & 0,85 \\
Méthionine $\quad 0,41$ & \\
Calcium & 0,925 \\
Phosphore & 0,38 \\
EM(kcal/kg) & 2702 \\
\hline
\end{tabular}




\section{DISCUSSION}

\section{Caractéristiques sociodémographiques des aviculteurs}

L'étude révèle que la majorité des fermiers sont des adultes avec un âge moyen qui tourne autour de 50 ans. Une majeure partie (80\%) des aviculteurs a été l'école. Ces résultats sont légèrement supérieurs à ceux de Teno (2010) qui a trouvé à Dakar (Sénégal) des fermiers dont l'âge moyen équivaut à 47 ans avec une forte prédominance des non instruits. Par ailleurs, il est remarqué dans l'échantillon $\mathrm{du}$ présent travail, une forte présence des fonctionnaires de l' 'Etat qui exercent le métier. L'aviculture est une activité lucrative qui génère des revenus. À l'inverse, il s'agit d'un domaine qui nécessite beaucoup d'investissement. Ce qui pourrait expliquer cette forte proportion de fonctionnaires. De plus, tous les fermiers enquêtés affirmaient qu'ils ont embarrassé ce métier à cause des revues tirés de la vente des œufs. Cette observation a été faite par Sonaiya et al. (2004) chez $89,2 \%$ des éleveurs qui indiquent la vente comme l'unique raison pour laquelle ils s'adonnent à l'aviculture. Pour la situation matrimoniale, tous les aviculteurs sont des mariés. Cette proportion est légèrement supérieure à celle de (Teno, 2010) qui a obtenu $88,2 \%$ des mariés au cours de ses travaux.

\section{Caractéristique de l'habitat des pondeuses}

L'étude a permis de constater que $70 \%$ des enquêtés ont en moyenne deux bâtiments sans aucun respect des normes techniques en rapport avec la distance inter-bâtiments. Ce résultat corrobore à celui d'Idi et Ganda (2005) qui ont indiqué que la distance minimale de séparation de $30 \mathrm{~m}$ entre les poulaillers. Concernant les dimensions particulièrement, les résultats obtenus au cours de cette étude sont similaires à ceux obtenus par Maizama et al. (2003) où les fermes avicoles ont une superficie variant de 142 à $150000 \mathrm{~m}^{2}$.

La conception d'un bâtiment d'élevage et l'ambiance qui règne dans ce dernier (bâtiment) sont des indicateurs clés pour assurer une bonne production. L'orientation de l'axe principal du bâtiment dans la direction des vents violents protègent les animaux qui $\mathrm{y}$ vivent. La régulation de la température permet une meilleure croissance des poussins et même les poules. En effet, chez les fermes avicoles de Niamey et Tillabéri, il est observé que dans $80 \%$ des fermes suivies, les bâtiments ont des faitières et $100 \%$ sont dotées d'une ventilation statique ou naturelle, un chauffage à charbon et l'éclairage naturelle. Ces observations ont été faites par Idi et Ganda (2005). La conception des bâtiments sans faitière, un toit ne débordant pas du tout ou pas assez pour limiter la pénétration des rayons du soleil et des eaux de pluie, ne pourraient pas assurer une ventilation optimale (Idi et Ganda, 2005). Le non-respect des dimensions (longueur/largeur) constitue également un handicap pour la survie des poulets.

\section{Alimentation}

Pour une meilleure rentabilité de l'élevage des poules, il est obligatoire de mettre en place un équilibre alimentaire qui s'adapte aux races de poules et au type d'élevage Drogoul et al., 2004; Laitat, 2004; Houndonougbo et al., 2012a; Hien et al., 2017). Il convient d'abord de préciser l'existence de différences de régime entre les poules en liberté et les poules en poulaillers. Pour celles en liberté, l'intervention de l'éleveur est réduite, car la poule mange ce dont elle a besoin, trouvé dans la nature. Il est remarqué que la disponibilité de quelques aliments essentiels tels que les protéines, les vitamines et les minéraux varie selon les saisons et les conditions atmosphériques à savoir l'humidité et la sècheresse (Lapierre et Pressenda, 2002). En ce qui concerne les poules au poulailler, l'éleveur est appelé à contrôler le régime nutritionnel de ses poules. Pour cela, il doit avoir une parfaite connaissance des besoins de ses volailles qui varient suivant l'âge, la race et le type d'élevage (poules de chair ou poules pondeuses)

\section{Soins sanitaires et prophylactiques}

Au cours du présent travail, il est observé une grande défaillance au plan sanitaire. En effet, la forte proportion (90\%) des fermiers obtenus lors de cette étude pratiquant irrégulièrement la désinfection 
(bâtiments et matériels) n'est pas reluisante. Pour corroborer, Idi et Ganda (2005) ont fait la même observation et ont indiqué qu'en général le niveau d'hygiène est en deca des normes de l'aviculture intensive.

En général, le bien être du vivant dépend en grande partie de son environnement (ITAVI et al., 2002; Laitat, 2004 ; Borda, 2007; Rés'OGM Info, 2008). Tous les répondants $(100 \%)$ font face à de nombreuses difficultés dans la mesure où ils ne maitrisent pas le domaine à l'entame de cette activité. Ces résultats sont similaires à celle de (Maizama et al. (2003) qui affirment qu'une ferme sur deux bénéficie du suivi d'un technicien avicole les premières semaines au démarrage de bande et lorsque surviennent de grosses mortalités.

Bien que l'élevage industriel de la poule relève de l'histoire contemporaine, il est reconnu qu'elle demeure l'animal domestique le plus manipulé artificiellement par l'homme (Granevitze et al., 2007). Par ailleurs, des études ont permis d'une part de connaitre son alimentation (Kondombo et al., 2003; Pousga et al., 2005; Ayssiwede et al., 2013; Nahimana et al., 2016), et d'autre part, de maitriser les méthodes d'élevage (Alders, 2005; Fotsa et al., 2007; Missohou et al., 2010), de reproduction (Msoffe et al., 2004; Akouango et al., 2010) et de sélection (Coquerelle, 2000; Beaumont et Chapuis, 2004). En dépit de cela, il est remarqué des points obscurs sur l'histoire de la poule même si certains d'entre eux furent éclaircis ces dernières années par des recherches effectuées sur l'espèce (Bismwa, 2003; Hosson, 2010).

\section{Conclusion}

Il convient de rappeler que ce travail a pour objet d'étudier les pratiques de l'élevage au sol des poules pondeuses. Globalement, cette étude basée sur une enquête a permis, certes, de remarquer un progrès remarquable dans la filière avicole. Toutefois, il est relevé au cours de ce travail des nombreuses insuffisances. Pour la conception de l'habitat, il est remarqué un non-respect des dimensions, des erreurs ont été repérées sur la construction des bâtiments, dans le système d'aération ainsi que la ventilation et l'éclairage. Pour l'alimentation, la majeure partie des fermiers achète l'aliment complet préparé. Enfin, au plan sanitaire, l'analyse des données fait ressortir que la vaccination et la désinfection des bâtiments sont les pratiques auxquelles font recours ces fermiers pour protéger leurs animaux. Au regard de ces contraintes, la présente suggère d'autres investigations dans l'avenir.

\section{INTERETS CONCURRENTS}

Les auteurs déclarent qu'ils n'ont pas d'intérêts concurrents".

\section{CONTRIBUTIONS DES AUTEURS}

HA et BRA ont participé à la conception et la planification de l'étude. Ils ont participé également à la rédaction de la première version du manuscrit. HA a analysé les données et a révisé toutes les versions du manuscrit. $\mathrm{AL}$ a révisé la dernière version du manuscrit. Il a bien contribué à l'amélioration de l'analyse statistique.

\section{REMERCIEMENTS}

Les auteurs remercient vivement les personnes qui ont contribuées pour la réussite de la présente étude.

\section{REFERENCES}

Alders R. 2005. L'aviculture : source de profit et de plaisir. Organisation des Nations Unies pour l'alimentation et l'agriculture: Rome.

Akouango FC. 2010. Croissance pondérale et productivité de la poule locale Gallus domesticusen élevage fermier au Congo. Anim. Genet. Resour., 46: 61-65.

Ayssiwede SB, Dieng A, Houinato MR, Chrysostome CAM, Issa Y, Hornick J.-L, Missohou A. 2013. Elevage des poulets traditionnels ou indigènes au Sénégal et en Afrique subsaharienne : état des lieux et contraintes. Ann. Méd. Vét., 157: 103119.

Beaumont C, Chapuis H. 2004. Génétique et sélection avicole : évolution des méthodes et des caractères. INRA Prod. Anim., 17 (1): 35-43. 
Bisimwa C. 2003. Les principales races en aviculture. Troupeaux et Cultures des Tropiques, Dossier spécial volaille, 1, 48.

Bordas A. 2007. Exploitation de la poule locale (Gallus gallus) en zone de forêt humide du Cameroun. Bull. Anim. Health. Afr., 55: 59-73p.

CILSS, CEDEAO, MRA, MAHRH, FEWS, NETIUSAID. 2006. Impacts socioéconomiques de la grippe aviaire en Afrique de l'Ouest : «Etude de cas au Burkina Faso » Draft. Burkina Faso.

Coquerelle G. 2000. Les Poules : Diversité Génétique Visible. Collection du Labo au Terrain, INRA Editions: Paris.

Dahouda M, Toléba SS, Senou M, Youssao AKI, Hambuckers A, Hornick J.-L. 2009. Les ressources alimentaires nonconventionnelles utilisables pour la production aviaire en Afrique : Valeurs nutritives et contraintes. Ann Med. Vet, 153: 5-21.

Drogoul C, Gadour R, Joseph M, Jussiau R. 2004. Nutrition et Alimentation des Animaux d'Elevage. Edit. Educagri: Dijon.

Dronne Y. 2019. Les matières premières agricoles pour l'alimentation humaine et animale : l'UE et la France. INRA Productions Animales., 31 (3): 181-200. DOI :

https://doi.org/10.20870/productionsanimales.2018.31.3.2347.

FAOSTAT. 2012. Données statistiques de la FAO (FAO-STAT). 08 Novembre, 2012, from

http://countrystat.org/home.aspx?c=BEN $\& \mathrm{tr}=7$.

Fotsa JC, Rognon X, Tixierboichard M, Ngou Ngoupayou JD, Pone Kamdem D, Manjeli Y, Granevitze Z, Hillel J, Chen GH, Cuc NT, Feldman M, Eding H, Weigend S. 2007. Genetic diversity within chicken populations from different continents and management histories. Animal Genetics, 38(6): 576-583. DOI: 10.1111/j.1365-2052.2007.01650.x •

Hien OC, Diarra B, Coulibaly Y. 2017. Effets d'une ration à base de la variété de maïs «
Espoir » sur la productivitédes poulettes. Int. J. Biol. Chem. Sci., 11(2): 806-816.

Houndonougbo MF, Chrysostome CAAM, Houndonougbo VP. 2012a. Performances bioéconomiques des poulettes alimentées avec des rations à base de feuilles séchées de manioc (Manhiote sculenta). Int. J. Biol. Chem. Sci., 6(2): 670-676.

Houndonougbo MF, Chrysostome CAAM, Houndonougbo VP. 2012b. Performances de ponte et qualité des œufs des poules pondeuses ISA Brown alimentées avec des rations à base de feuilles séchées de manioc (Manhiotesculenta). Int. J. Biol. Chem. Sci., 6(5): 1950-1959.

Hossen MJ. 2010. Effect of management intervention on the productivity and profitability of indigenous chickens under rural condition in Bangladesh. Livest. Res. Rural Dev., 22: 192.

Idi A, Ganda I. 2005. Etat actuel du bâtiment et du matériel d'élevage avicole dans la zone d'intervention du Projet de Sécurisation de l'Elevage et de l'Agriculture périurbains de Niamey. Document technique, PSEAU: Niamey.

INS. 2018. Annuaire Statistique Du Niger, 2013 - 2017. 260p.

Issa S, Hancok JD, Tunista MR., Brah N, Hassan A, Kapran I, Kaka S. 2010. Le sorgho un bon substitut dans l'alimentation des poulets de chair. Communication en Aviculture Familiale, 19 (1): 16-22.

ITAVI, AFSSA, CIRAD. 2002. La production d'œufs en climat chaud. Ed. ITAVI.

Kondombo SR, Kwakkel RP, Nianogo AJ, Slingerland M. 2003. Effects of local feed stuff supplementation on performance and nutritional status of village chickens during the end of the rainy season in Burkina Faso. Rev. Elev. Med. Vet. Pays Trop., 56: 199-204.

Laitat M. 2004. Facteurs influençant la consommation alimentaire et les performances zootechniques du porc sevré : perception et caractéristiques de l'aliment. Ann. Méd. Vét., 148: 15-29.

Lapierre O, Pressenda F. 2002. Adaptation des stratégies d'approvisionnement des 
filières animales en matières premières riches en protéines. OCL, 9: 86-91.

Maikano I, Alassane, A. 2006. Situation de la grippe aviaire au Niger. Communication présentée à l'atelier "Impact de l'Influenza aviaire sur l'avicultre à petite échelle en Afrique de l'Ouest : nécessité d'une collaboration régionale" Tamale, Ghana, 12-14 Septembre 2006.

Maizama DG, Sanoko F, Ganahi, A. 2003. Repères pour un développement de la filière avicole moderne au Niger, Rapport final, deuxième version, Direction des Etudes et de la Programation, Ministère des Ressources Animales.

Missohou A, Soumboudou A, Ayssiwede BS, Hane M. 2010. Mise au point de poussinière pour améliorer la productivité du couple mère-poussins en aviculture familiale au Sénégal. In: Forum national de la recherche scientifique et des innovations technologiques, Bobo Dioulasso, Burkina Faso, 27 nov. - 4 déc. 2010.

Msoffe PLM, Mtambo MMA, Minga UM, Olsen JE, Juul-Madsen HR, Gwakisa PS, Mutayoba SK, Katule AM. 2004. Productivity and reproductive performance of the free-range local domestic fowl ecotypes in Tanzania. Livest. Res. Rural Dev., 16 (9). http://www.lrrd.org/lrrd16/9/msof16067. htm.

Nahimana G, Missohou A, Ayssiwede SB., Cissé P, Butore J, Touré A. 2017. Amélioration de la survie des poussins et des performances zootechniques de la poule locale en condition villageoise au
Sénégal. Rev. Elev. Med. Vet. Pays Trop., 70 (1): 3-8. DOI: 10.19182/remvt.31393. Nworgu FC, Fasoghon FO. 2007. Centrosema (Centrosema pubescens) leaf meal as protein supplement for pullet chicks and growing pullets. Int. J. Biol. Chem. Sci., 6(4): 255-260.

Ouedraogo CL. 2003. Rôle et place du petit élevage dans la lutte contre la pauvreté, Version provisoire, Ministère des Ressources Animales, Burkina Faso, p14. République du Niger, Ministère de la population. 2012. Recensement Général de la Population et de l'Habitat.

Pousga S, Boly H, Linderberg JE, Ogle B. 2005. Scavenging pullets in BurkinaFaso: Effect of season, locationand breed on feed and nutrientintake. Trop. Anim. Health Prod., 37: 623-634.

Rés'OGM Info. 2008. Guide pour une agriculture durable, innovante et sans OGM Liens Un article d'Arvalis sur les moyens de lutte contre les mycotoxines sur maïs et blé. http://www.arvalisinstitutduvegetal.fr/fr/ arvalis_infos/numero2/p8-9.pdf.

Sonaiya EB, Swan SEJ. 2004. Production en Aviculture Familiale, un Manuel Technique. Organisation des Nations Unies pour l'Alimentation et l'Agriculture: Rome.

Teno G. 2010. Analyse du système de commercialisation du poulet du pays dans le département de Dakar (Sénégal). Mémoire de master II, Ecole Inter-états des Sciences et Médecine Vétérinaire de Dakar, Dakar, p. 31. 